\title{
Position Constrained Bilateral Control by Oblique Coordinate Control Considering Priority of Tasks
}

\author{
Sho Sakaino* Member, Tomoya Sato** Non-member

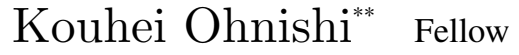

(Manuscript received Nov. 10, 2012, revised July 4, 2013)

\begin{abstract}
This paper proposes two position constrained bilateral controllers. The constraint is given by coordinate transformation and additional compensator or command modification methods are not necessary. The constraint therefore does not destabilize the system and maintains linearity. The difference between the two proposed methods is the task priority. The first ensures slave position limitation and force regulation between two robots, the other ensures position limitation and position regulation between the robots. We analyzed the stability of the proposed controller. The validity of the proposed methods was confirmed experimentally.
\end{abstract}

Keywords: bilateral control, oblique coordinate control, haptics, position constraint, constrained system

\section{Introduction}

Recently, robots are expected to work around human environments. Welfare robots, medical robots, patrol robots, and many human-friendly applications are keeping our attention. When robots move around us, guarantee of safety is one of the most important issues. However, conventional manufacturing robots do not have an ability to avoid dangerous situations. Instead of that, operators should care about moving range of robots. Thus, the conventional robots impose hard work on us and, obviously, they are not human-friendly. Therefore, robots around human environments themselves should assure safety.

In this research, we focus on constrained bilateral control. Bilateral control gives remote manipulation of a slave (remote) robot by a master (local) robot. At the same time, an operator can feel the reaction force of the slave. By this controller, an operator recognizes the remote environment as if it is existed in the local side. One interesting application of this technique is robotic surgery. A doctor manipulates the master and a patient is operated on by the slave. Robotic surgery realizes many useful applications such as minimally invasive surgery ${ }^{(1)-(3)}$, brain surgery ${ }^{(4)}$, cell manipulation $^{(5)-(7)}$, and telesurgery ${ }^{(8)(9)}$. Hannaford and Lawrence introduced indices to evaluate the performance of bilateral controllers $^{(10)(11)}$. Many kinds of bilateral controllers have been proposed ${ }^{(12)-(14)}$. However, the performance of them is not sufficient. Yokokohji et al. proposed a precise bilateral

\footnotetext{
${ }^{*}$ Department of Electrical and Electronic Systems, Faculty of Engineering, Saitama University

255, Shimo-Ohkubo, Sakura-ku, Saitama City, Saitama 3388570, Japan

** Department of System Design Engineering, Faculty of Science and Technology, Keio University

3-14-1, Hiyoshi, Kohoku-ku, Yokohama City, Kanagawa 2238522, Japan
}

controller and showed that bilateral controllers are based on two tasks ${ }^{(15)}$; position difference of the master and the slave should be zero and the force summation of the master and the slave should be zero. The second task indicates the law of action and reaction. Matsumoto et al. proposed "4-channel bilateral controller" and improved the performance of the Yokokohjis' method ${ }^{(16)}$ by exploiting a disturbance observer (DOB) ${ }^{(17)}$. As it is mentioned, however, the slave robot itself should assure safety to avoid serious accidents. Otherwise, patients will be damaged. Constraints of the slave are hence necessary.

Most of position constraint methods are divided into two directions. The first is a constraint by a nonlinear controller ${ }^{(18)(19)}$. The other is a constraint by modification of input commands ${ }^{(20)(21)}$. However, these methods require deep knowledge of plants; robots, humans, and environments, making it difficult to obtain ideal constrained systems. Therefore, systems under these constraints tend to be conservative, to be unstable, and to be difficult to describe the interference of tasks. Some researchers studied constrained bilateral control based on each of the constraints ${ }^{(22)(23)}$. Hence, inherently they cannot obtain ideal constrained bilateral controllers. The basic problem of the conventional constraints is "difficulty of designing" because the constraints strongly connected with plants. The conventional method ${ }^{(22)}$ needs additional stability analysis for position constraints. However, even though brief analysis of the controller is explained in the literature ${ }^{(22)}$, it is not sufficient because the entire system is nonlinear and is difficult to analyze. The method shown in (23) does not have a force controller, because position constrained force controllers are difficult to design. The designing obviously gets difficult according to the system complexity. Needless to say, humans and human environments are quite complicated plants making the conventional methods unable to be adapted. To solve the problem, the designing of constraints must be obtained independently of plants. 
Recently, we found a way to design tasks independently of plants ${ }^{(24)}$. Because the method oblique coordinate control treats tasks as coordinate transform (kinematics), the controller designing, which are given based on plants (dynamics), can be obtained without considering tasks. Once a stable system is obtained, then, a system after transform; a system for certain tasks, is also stable. This feature makes combinations of tasks easy since it can be translated into sum and multiple of coordinate transform matrices. Hence, bilateral control, which is a combination of position and force tasks, was achieved with few designing efforts. We also found that position constraints can be obtained by coordinate transform and stable position constrained bilateral control was achieved ${ }^{(25)}$. If a position of a slave passes through a threshold value (the slave is in a dangerous area), the coordinate transform changes its form and keeps the slave in an adequate area. When the slave is in the dangerous area, however, the controller cannot realize both of the tasks of bilateral control (position tracking and force regulation).

A bilateral control system has two degrees of freedom (DOF): the single-DOF master and the single-DOF slave. Note that DOF of the control system means the sum of DOF of the master and slave robots. This implies that even though the slave is in the dangerous area, and one DOF is used for the constraint, there is still one DOF remaining. If we utilize the remaining DOF, each of the bilateral control tasks can be realized. We propose two constrained bilateral controllers that utilize the remaining DOF. One controller regulates the force summation to zero. The other regulates the position difference to zero. Since these controllers are created based on oblique coordinate control, designers can realize a precise constrained bilateral controller without caring destabilization by the constraints. In addition, since the stability of oblique coordinate control based position constrained systems have not been explicitly shown yet, the stability is also discussed in this paper. Its validity is experimentally verified.

There may be better position constraint methods than the proposed method in terms of the tracking performance and the stability. We do not insist that the proposed method has the highest performance and is most stable. We want to create "plug-and-play controllers." It means that designers can add and delete a new function (task) to an existing stable controlled system with few efforts. This is an originality of an oblique coordinate control based task designing approach.

This paper is composed of 7 parts. A robot model used in this research is shown in section 2. In section 3, coordinate transform matrices for the proposed constrained bilateral controllers are derived. A controller is shown in section 4. The stability of the position constraint is discussed in section 5 . The validity is experimentally verified in section 6 . In the last section, a conclusion of this research is given.

\section{Robot Model}

Fig. 1 shows master and slave robots. Fig. 2 is their simplified models. $x, f, m$ are position, force, and mass. Variables with subscript 1 is that of the master, and variables with subscript 2 is that of the slave. Their dynamics is given as follows:

$$
\boldsymbol{m} \ddot{\boldsymbol{x}}^{\text {res }}=\boldsymbol{f}^{\text {input }}-\boldsymbol{f}^{\text {res }} .
$$

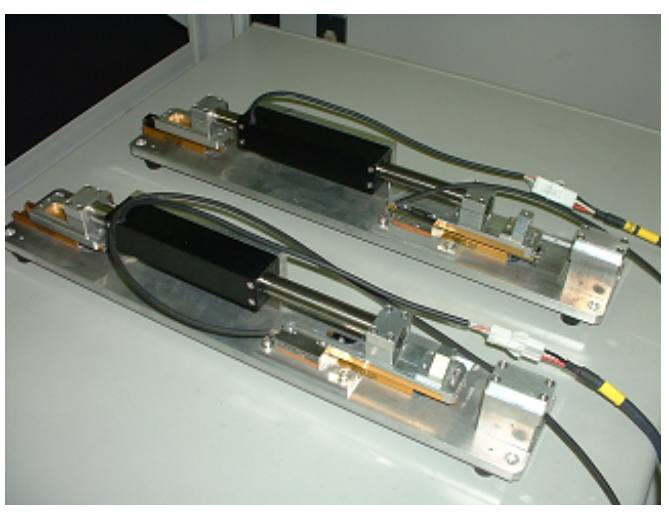

Fig. 1. Robot
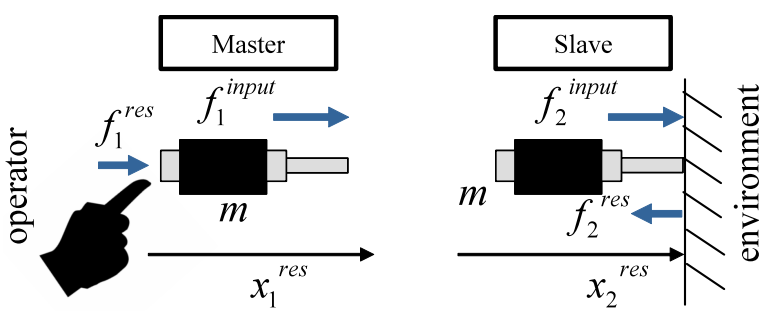

Fig. 2. Robot model

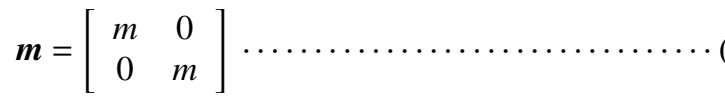

$$
\begin{aligned}
& \boldsymbol{x}=\left[\begin{array}{l}
x_{1} \\
x_{2}
\end{array}\right], \boldsymbol{f}=\left[\begin{array}{l}
f_{1} \\
f_{2}
\end{array}\right] .
\end{aligned}
$$

Variables with res are responses and those with input are control inputs.

\section{Coordinate Transform for Constrained Bilateral Control}

Oblique coordinate control can give variety of tasks only by definition of coordinate transform (task Jacobian matrix $)^{(24)}$. The space after the transform is called "modal space." In this section, two coordinate transforms: Constraint 1 and Constraint 2, are proposed.

3.1 Position Limitation Fig. 3 shows a schematic view of a position limitation coordinate. $\hat{x}_{2}$ is a virtual position and $x_{\text {lim }}$ is a limitation value. Here, $a$ is a threshold value and is an arbitrary tuning parameter. The coordinate should satisfy the following requirements.

- $x_{2}<x_{\text {lim }}$ is always satisfied.

- $\hat{x}_{2}=x_{2}$ should be satisfied in a safe area $\left(x_{2}<a\right)$.

- $\hat{x}_{2}$ and $\dot{\hat{x}}_{2}$ should be continuous.

- $\hat{x}_{2}$ is a monotonically increasing function of $x_{2}$.

The first requirement means that $x_{2}$ is saturated. The second requirement means that the constraint does not deteriorate the response when the position is in the safe area. The third requirement assures the existence of a Jacobian matrix. The fourth requirement is needed for the stability. If we control $\hat{x}_{2}$ instead of $x_{2}, x_{2}$ never reaches to a position limitation value $x_{\text {lim }}$. The greater $\left|x_{\text {lim }}-a\right|$ is, the more conservative the system becomes.

In this paper, to meet the requirements, we choose an exponential function as a representative of saturating functions. Other saturating functions such as arc tangent functions (in 


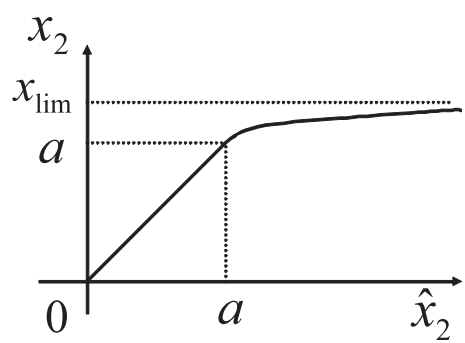

Fig. 3. Position limitation coordinate

this case the second requirement is relaxed as $\hat{x}_{2} \approx x_{2}$ ) can be adaptable. Then, an exponential function that satisfies the requirements are described in (4) and (5).

$$
\begin{aligned}
& x_{2}=g\left(\hat{x}_{2}\right) \\
& = \begin{cases}\hat{x}_{2} & \left(x_{2}<a\right) \\
\left(x_{\text {lim }}-a\right)\left(1-e^{-b\left(\hat{x}_{2}-a\right)}\right)+a & \left(x_{2} \geq a\right)\end{cases} \\
& \dot{x}_{2}= \begin{cases}\dot{\hat{x}}_{2} & \left(x_{2}<a\right) \\
e^{-b\left(\hat{x}_{2}-a\right)} \dot{\hat{x}}_{2} & \left(x_{2} \geq a\right)\end{cases} \\
& b=\frac{1}{x_{\text {lim }}-a}
\end{aligned}
$$

Its inverse function is given as follows:

$$
\begin{aligned}
& \hat{x}_{2}=g^{-1}\left(x_{2}\right) \text {. } \\
& = \begin{cases}x_{2} & \left(x_{2}<a\right) \\
\left(a-x_{\text {lim }}\right) \ln \left(\frac{x_{\text {lim }}-x_{2}}{x_{\text {lim }}-a}\right)+a & \left(x_{2} \geq a\right)\end{cases} \\
& \dot{\hat{x}}_{2}= \begin{cases}\dot{x}_{2} & \left(x_{2}<a\right) \\
e^{b\left(\hat{x}_{2}-a\right)} \dot{x}_{2} & \left(x_{2} \geq a\right)\end{cases}
\end{aligned}
$$

The slave position is then constrained in (10).

$$
\begin{aligned}
& \dot{\hat{x}}_{2}=\boldsymbol{J}_{\text {lim }}\left[\begin{array}{l}
\dot{x}_{1} \\
\dot{x}_{2}
\end{array}\right] \ldots \ldots \ldots \ldots \ldots \ldots \ldots \\
& \boldsymbol{J}_{\text {lim }}= \begin{cases}{\left[\begin{array}{ll}
0 & 1
\end{array}\right]} & \left(x_{2}<a\right) \\
{\left[\begin{array}{ll}
0 & e^{b\left(\hat{x}_{2}-a\right)}
\end{array}\right]} & \left(x_{2} \geq a\right)\end{cases}
\end{aligned}
$$

$\boldsymbol{J}_{\text {lim }}$ is a task Jacobian matrix, which gives the constraint. Note that force $f_{2}$ is also transformed to the new force $\hat{f}_{2}$.

$$
\hat{f}_{2}=\boldsymbol{J}_{\text {lim }}\left[\begin{array}{l}
f_{1} \\
f_{2}
\end{array}\right] \text {. }
$$

Likewise, if we control $\hat{f}_{2}$ instead of $f_{2}$, this controller never violates the limitation.

3.2 Bilateral Control Bilateral control is composed of two tasks. The first is that the difference of the position of the master and the slave should be zero. This task gives position tracking.

$$
\begin{aligned}
& x_{1}-x_{2}=0 \ldots \ldots \\
& \boldsymbol{J}_{\boldsymbol{X}}\left[\begin{array}{c}
\dot{x}_{1} \\
\dot{x}_{2}
\end{array}\right]=0 \ldots \\
& \boldsymbol{J}_{\boldsymbol{X}}=\left[\begin{array}{ll}
1 & -1
\end{array}\right]
\end{aligned}
$$

The second task is that the summation of the force of the master and the slave should be zero. This task shows the law of action and reaction.

$$
\begin{aligned}
& \boldsymbol{J}_{\boldsymbol{F}}\left[\begin{array}{l}
f_{1} \\
f_{2}
\end{array}\right]=0 \cdots \\
& \boldsymbol{J}_{\boldsymbol{F}}=\left[\begin{array}{ll}
1 & 1
\end{array}\right] .
\end{aligned}
$$

$\boldsymbol{J}_{\boldsymbol{X}}$ and $\boldsymbol{J}_{\boldsymbol{F}}$ are task Jacobian matrices for the position task and the force task, respectively.

3.3 Constraint 1 Oblique coordinate control easily gives hierarchy of tasks by a multiple of task Jacobian matrices. In this case, a matrix multiplied from the right side is a primal task.

Here, Constraint 1 is proposed. It limits the slave position and, at the same time, the force regulation is always achieved.

- The primal tasks are $\boldsymbol{J}_{\text {lim }}$ and $\boldsymbol{J}_{\boldsymbol{F}}$.

- The secondary task is $\boldsymbol{J}_{\boldsymbol{X}}$.

The primal task matrix $\boldsymbol{J}_{\text {prime } 1}$ is given as follows:

$$
\boldsymbol{J}_{\text {prime } 1}=\left[\begin{array}{c}
\boldsymbol{J}_{\text {lim }} \\
\boldsymbol{J}_{\boldsymbol{F}}
\end{array}\right]
$$

A task Jacobian matrix for Constraint $1 \boldsymbol{J}_{\boldsymbol{t} \mathbf{1}}$ is then given as follows:

$$
\boldsymbol{J}_{\boldsymbol{t} \mathbf{1}}=\boldsymbol{J}_{\text {second } 1} \boldsymbol{J}_{\text {prime } 1}
$$

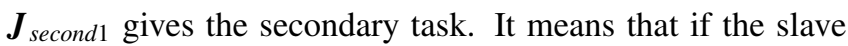
robot is in safe area such that $x_{2}<a, \boldsymbol{J}_{t 1}$ should satisfy the next equation.

$$
\boldsymbol{J}_{\boldsymbol{t} \mathbf{1}}=\left[\begin{array}{l}
\boldsymbol{J}_{\boldsymbol{X}} \\
\boldsymbol{J}_{\boldsymbol{F}}
\end{array}\right] \quad\left(x_{2}<a\right)
$$

$\boldsymbol{J}_{\text {second } 1}$ is thus obtained as follows:

$$
\begin{aligned}
\boldsymbol{J}_{\text {second } 1} & =\left[\begin{array}{c}
\boldsymbol{J}_{X} \\
\boldsymbol{J}_{\boldsymbol{F}}
\end{array}\right] \boldsymbol{J}_{\text {prime } 1}^{-1}\left(x_{2}<a\right) \\
& =\left[\begin{array}{cc}
-2 & 1 \\
0 & 1
\end{array}\right] \ldots \ldots \ldots \ldots \ldots
\end{aligned}
$$

The task Jacobian matrix for Constraint 1 is then obtained.

$$
\boldsymbol{J}_{\boldsymbol{t} \mathbf{1}}= \begin{cases}{\left[\begin{array}{cc}
1 & -1 \\
1 & 1
\end{array}\right]} & \left(x_{2}<a\right) \\
{\left[\begin{array}{cc}
1 & -2 e^{b\left(\hat{x}_{2}-a\right)}+1 \\
1 & 1
\end{array}\right]} & \left(x_{2} \geq a\right)\end{cases}
$$

3.4 Constraint 2 Next, Constraint 2 is proposed. It limits the slave position and, at the same time, the position tracking is always achieved.

- The primal tasks are $\boldsymbol{J}_{\text {lim }}$ and $\boldsymbol{J}_{\boldsymbol{X}}$.

- The secondary task is $\boldsymbol{J}_{\boldsymbol{F}}$.

The primal tasks are given by a task Jacobian matrix $\boldsymbol{J}_{\text {prime } 2}$.

$$
\boldsymbol{J}_{\text {prime } 2}=\left[\begin{array}{c}
\boldsymbol{J}_{\boldsymbol{X}} \\
\boldsymbol{J}_{\text {lim }}
\end{array}\right] .
$$

A task Jacobian matrix for Constraint $2 J_{t 2}$ is then given as follows:

$$
\boldsymbol{J}_{\boldsymbol{t} \mathbf{2}}=\boldsymbol{J}_{\text {second } 2} \boldsymbol{J}_{\text {prime } 2}
$$

$\boldsymbol{J}_{\text {second } 2}$ is therefore obtained as follows: 


$$
\begin{aligned}
\boldsymbol{J}_{\text {second } 2} & =\left[\begin{array}{l}
\boldsymbol{J}_{X} \\
\boldsymbol{J}_{\boldsymbol{F}}
\end{array}\right] \boldsymbol{J}_{\text {prime }^{-1}} \quad\left(x_{2}<a\right) \ldots \ldots \ldots \\
& =\left[\begin{array}{ll}
1 & 0 \\
1 & 2
\end{array}\right] \ldots \ldots \ldots \ldots \ldots \ldots \ldots \ldots
\end{aligned}
$$

Finally, the task Jacobian matrix for Constraint 2 is obtained.

$$
J_{t \mathbf{2}}= \begin{cases}{\left[\begin{array}{cc}
1 & -1 \\
1 & 1
\end{array}\right]} & \left(x_{2}<a\right) \\
{\left[\begin{array}{cc}
1 & -1 \\
1 & 2 e^{b\left(\hat{x}_{2}-a\right)}-1
\end{array}\right]} & \left(x_{2} \geq a\right)\end{cases}
$$

\section{Oblique Coordinate Control}

The velocity and force in the modal space, $\dot{\boldsymbol{X}}$ and $\boldsymbol{F}$, are given as follows:

$$
\begin{aligned}
\dot{X} & =\left[\begin{array}{c}
\dot{X}_{X} \\
\dot{X}_{F}
\end{array}\right]=J_{t} \dot{x} \\
\boldsymbol{F} & =\left[\begin{array}{c}
F_{X} \\
F_{F}
\end{array}\right]=\boldsymbol{J}_{\boldsymbol{t}} \boldsymbol{f} .
\end{aligned}
$$

Here, $\boldsymbol{J}_{\boldsymbol{t}}=\boldsymbol{J}_{\boldsymbol{t} \mathbf{1}}$ with Constraint 1 and $\boldsymbol{J}_{\boldsymbol{t}}=\boldsymbol{J}_{\boldsymbol{t} \mathbf{2}}$ with Constraint 2. Note that $\boldsymbol{F}=\boldsymbol{J}_{\boldsymbol{t}}^{-T} \boldsymbol{f}$ is commonly used to transform the force vectors. In the modal transformation, position and force are transformed by the same matrix, and task design becomes easy. Obviously, the energies before and after the modal transformation differ. Then, positions in the modal space with each constraint are given as follows:

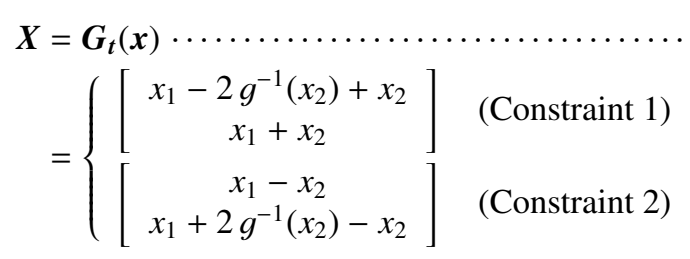

A modal mass matrix $\boldsymbol{M}$, which gives dynamic properties in the modal space, is given as follows ${ }^{(25)}$ :

$$
\boldsymbol{M}=\boldsymbol{J}_{\boldsymbol{t}} \boldsymbol{m} \boldsymbol{J}_{t}^{-1} \text {. }
$$

The dynamics in the modal space is derived by using the modal mass matrix ${ }^{(25)}$.

$$
\boldsymbol{M} \ddot{\boldsymbol{X}}^{\text {res }}=\boldsymbol{F}^{\text {input }}-\boldsymbol{F}^{\text {res }}+\boldsymbol{M} \dot{\boldsymbol{J}}_{\boldsymbol{t}} \dot{\boldsymbol{x}}^{\text {res }}
$$

Because $\boldsymbol{m}=m \boldsymbol{I}$ as it is shown in (2), (33) is simplified as

$$
\boldsymbol{M}=\boldsymbol{m} \text {. }
$$

Here, $\boldsymbol{I}$ is a unit matrix. In this research, the acceleration and force references are determined by a proportional-derivative controller and a proportional controller as follows:

$$
\begin{aligned}
& \ddot{X}^{r e f}=-C_{p} \boldsymbol{X}^{r e s} \ldots \ldots \ldots \\
& \boldsymbol{F}^{r e f}=-C_{f} \boldsymbol{F}^{r e s} \ldots \ldots \ldots \ldots \\
& C_{p}=K_{p}+K_{v} s, C_{f}=K_{f} .
\end{aligned}
$$

Variables with ref are references. Constants $K_{p}, K_{v}, K_{f}$ are position, velocity, and force feedback gains. In oblique coordinate control, acceleration and force references are unified by using a hybrid matrix $\boldsymbol{H}$, which is defined as follows:

$$
\begin{aligned}
& {\left[\begin{array}{c}
F_{X} \\
\ddot{X}_{F}
\end{array}\right]=\boldsymbol{H}\left[\begin{array}{c}
\ddot{X}_{X} \\
F_{F}
\end{array}\right] .} \\
& \boldsymbol{H}=\left[\begin{array}{cc}
m & 0 \\
0 & \frac{1}{m}
\end{array}\right] . \cdots
\end{aligned}
$$

Fig. 4 shows oblique coordinate control ${ }^{(25)}$. Here, $S$ shown in Fig. 4 is a position/force selection matrix defined as follows:

$$
S=\left[\begin{array}{ll}
1 & 0 \\
0 & 0
\end{array}\right]
$$

Sustituting (35), (36), (37), (40), and the task Jacobian matrix $\boldsymbol{J}_{t}$ for the controller, a position constrained bilateral controller is obtained.

$$
\boldsymbol{F}^{\text {input }}=\left[\begin{array}{c}
m \ddot{X}_{X}^{r e f} \\
F_{F}^{r e f}
\end{array}\right]-\boldsymbol{M} \dot{\boldsymbol{J}}_{t} \dot{\boldsymbol{x}}^{r e s} \ldots \ldots \ldots \ldots \ldots \ldots
$$

This force reference is transformed to inputs for actuators as follows:

$$
\boldsymbol{f}^{\text {input }}=\boldsymbol{J}_{t}^{-1} \boldsymbol{F}^{\text {input }}
$$

As it is shown above, designers need to concentrate only on the selection of position $\boldsymbol{X}=\boldsymbol{G}(\boldsymbol{t})$. The ways to derive the dynamics and to design the controller are not changed by $\boldsymbol{X}$. Note that $\boldsymbol{X}$ is just kinematics. Hence, large tasks, which are combinations of small tasks, are obtained by assembling kinematics of small tasks.

\section{Stability}

In this section, the stability of the proposed method is analyzed. First, we show that oblique coordinate control is equivalent to the 4-channel bilateral controller when there is no constraint. Then, the stability of oblique coordinate control with constraints is shown. The stability of the 4channel bilateral controller has been analyzed by many papers ${ }^{(16)(26)-(29)}$. Because they showed that practically the 4channel bilateral controller is stable, in this paper, we assume that oblique coordinate control without constraint is stable. Then, if we prove the effect of constraints does not destabilize the controller, then the stability of the position constrained bilateral controller by using oblique coordinate control is proved.

5.1 Oblique Coordinate Control and 4-channel Bilateral Control If there is no constraint, the task Jacobian

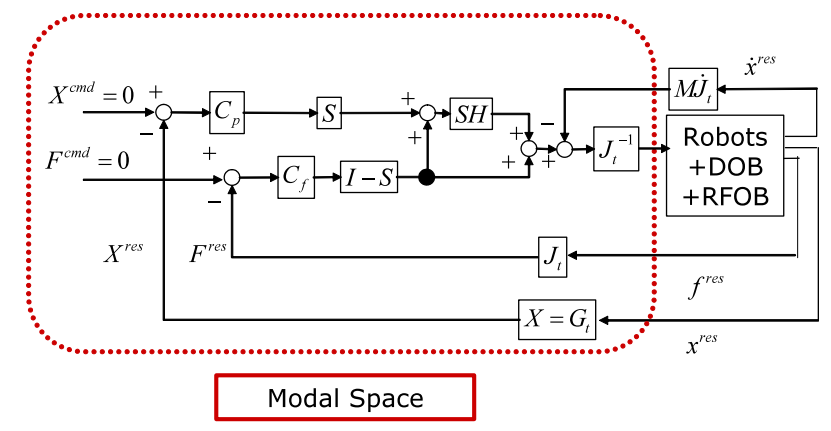

Fig. 4. Oblique Coordinate Control) 
matrix is given as follows:

$$
\boldsymbol{J}_{\boldsymbol{t}}=\left[\begin{array}{cc}
1 & -1 \\
1 & 1
\end{array}\right]
$$

Sustituting (44) for (42) and (43), a bilateral controller is obtained.

$$
\begin{aligned}
\boldsymbol{F}^{\text {input }} & =\left[\begin{array}{c}
m \ddot{X}_{X}^{r e f} \\
F_{F}^{r e f}
\end{array}\right] \ldots \ldots \ldots \ldots \\
\boldsymbol{f}^{\text {input }} & =\boldsymbol{J}_{\boldsymbol{t}}^{-1}\left[\begin{array}{c}
m \ddot{X}_{X}^{r e f} \\
F_{F}^{r e f}
\end{array}\right] \\
& =\frac{1}{2}\left[\begin{array}{c}
m \ddot{X}_{X}^{r e f}+F_{F}^{r e f} \\
-m \ddot{X}_{X}^{r e f}+F_{F}^{r e f}
\end{array}\right] .
\end{aligned}
$$

By defining $\ddot{\boldsymbol{x}}^{r e f}$ in (47), control inputs in acceleration dimension are obtained in (48).

$$
\begin{aligned}
\ddot{\boldsymbol{x}}^{r e f} & =\left[\begin{array}{c}
\ddot{x}_{1}^{r e f} \\
\ddot{x}_{2}^{\text {ref }}
\end{array}\right]=\boldsymbol{m}^{-1} \boldsymbol{f}^{\text {input }} \ldots \ldots \ldots \ldots \ldots \ldots \ldots \\
& =\frac{1}{2}\left[\begin{array}{c}
C_{p}\left(x_{2}^{r e s}-x_{1}^{r e s}\right)-\frac{C_{f}}{m}\left(f_{1}^{r e s}+f_{2}^{r e s}\right) \\
C_{p}\left(x_{1}^{r e s}-x_{2}^{r e s}\right)-\frac{C_{f}}{m}\left(f_{1}^{r e s}+f_{2}^{r e s}\right)
\end{array}\right] \ldots
\end{aligned}
$$

If we substitute $x_{1}, x_{2}, f_{1}, f_{2}, \frac{C_{f}}{m}$ for $x_{M}, x_{S}, f_{M}, f_{S}, C_{f}$, equation (48) is equal to equations (8) and (9) in the literature ${ }^{(30)}$. Note that the difference of $\frac{C_{f}}{m}$ and $C_{f}$ is trivial, because this is just a difference of feedback gain of a proportional controller. Therefore, we finally proved that oblique coordinate control without constraint is equal to the 4-channel bilateral controller.

5.2 Effect of Position Constraint Here we define a task Jacobian matrix $\boldsymbol{J}_{\boldsymbol{a}}$ that is not constrained. Then the dynamics with the task Jacobian matrix is derived as follows:

$$
\begin{aligned}
& \boldsymbol{M}_{\boldsymbol{a}} \ddot{\boldsymbol{X}}_{\boldsymbol{a}}{ }^{\text {res }}=\boldsymbol{F}_{\boldsymbol{a}}^{\text {input }}-\boldsymbol{F}_{\boldsymbol{a}}{ }^{\text {res }}+\boldsymbol{M}_{\boldsymbol{a}} \dot{\boldsymbol{J}}_{\boldsymbol{a}} \dot{\boldsymbol{x}}^{\text {res }} \\
& \dot{X}_{a}=J_{a} \dot{x} \\
& F_{a}=J_{a} f \\
& \boldsymbol{M}_{\boldsymbol{a}}=\boldsymbol{J}_{\boldsymbol{a}} \boldsymbol{m} \boldsymbol{J}_{\boldsymbol{a}}^{-1}
\end{aligned}
$$

Variables with subscript $\boldsymbol{a}$ means variables without constraints. Likewise, a constrained task Jacobian matrix $\boldsymbol{J}_{\boldsymbol{b}}$ is defined and the dynamics is derived.

$$
\begin{aligned}
& \boldsymbol{M}_{\boldsymbol{b}} \ddot{\boldsymbol{X}}_{\boldsymbol{b}}{ }^{\text {res }}=\boldsymbol{F}_{\boldsymbol{b}}{ }^{\text {input }}-\boldsymbol{F}_{\boldsymbol{b}}{ }^{\text {res }}+\boldsymbol{M}_{\boldsymbol{b}} \dot{\boldsymbol{J}}_{\boldsymbol{b}} \dot{\boldsymbol{x}}^{\text {res }} \ldots \ldots \ldots \\
& \dot{X}_{b}=J_{b} \dot{x} \\
& F_{b}=J_{b} f \\
& M_{b}=J_{b} \boldsymbol{m} J_{b}^{-1} \text {. }
\end{aligned}
$$

Variables with subscript $\boldsymbol{b}$ mean variables with constraints.

5.3 Position Control We assume that the following position controller stabilizes the position of the system (49).

$$
\boldsymbol{F}_{\boldsymbol{a}}^{\text {input }}=\boldsymbol{M}_{\boldsymbol{a}}\left(-C_{p} \boldsymbol{X}_{\boldsymbol{a}}{ }^{\text {res }}\right)+\boldsymbol{M}_{\boldsymbol{a}} \dot{\boldsymbol{J}}_{\boldsymbol{a}} \dot{\boldsymbol{x}}^{\text {res }}
$$

Substituting this input for (49), the position response is given as follows:

$$
\ddot{\boldsymbol{X}}_{\boldsymbol{a}}{ }^{\text {res }}=-C_{p} \boldsymbol{X}_{\boldsymbol{a}}{ }^{\text {res }}-\boldsymbol{F}_{\boldsymbol{a}}{ }^{\text {res }} .
$$

From the assumption, this position is stable. Then, a position controller for (53) is given as follows:

$$
\boldsymbol{F}_{\boldsymbol{b}}{ }^{\text {input }}=\boldsymbol{M}_{\boldsymbol{b}}\left(-C_{p} \boldsymbol{X}_{\boldsymbol{b}}{ }^{\text {res }}\right)+\boldsymbol{M}_{\boldsymbol{b}} \dot{\boldsymbol{J}}_{\boldsymbol{b}} \dot{\boldsymbol{x}}^{\text {res }} .
$$

The position response is given in (60).

$$
\ddot{\boldsymbol{X}}_{\boldsymbol{b}}{ }^{\text {res }}=-C_{p} \boldsymbol{X}_{\boldsymbol{b}}{ }^{\text {res }}-\boldsymbol{F}_{\boldsymbol{b}}{ }^{\text {res }} \text {. }
$$

Two equations (58) and (60) are identical. Furthermore, $\hat{x}_{2}$ is a monotonically increasing function of $x_{2}$. Then, obviously, the position of (60) is stable.

5.4 Force Control We assume that the following force controller stabilizes the force of the system (49).

$$
\boldsymbol{F}_{\boldsymbol{a}}{ }^{\text {input }}=-C_{f} \boldsymbol{F}_{\boldsymbol{a}}^{\text {res }}+\boldsymbol{M}_{\boldsymbol{a}} \dot{\boldsymbol{J}}_{\boldsymbol{a}} \dot{\boldsymbol{x}}^{\text {res }} .
$$

Substituting this input for (49), the next equation is derived.

$$
\boldsymbol{M}_{\boldsymbol{a}} \ddot{\boldsymbol{X}}_{\boldsymbol{a}}{ }^{\text {res }}=-\left(C_{f}+1\right) \boldsymbol{F}_{\boldsymbol{a}}{ }^{\text {res }}
$$

As it is mentioned above, the modal transformation does not keep the energy and there is a following relation obtained by (50) and (51).

$$
\boldsymbol{f}^{T} \dot{\boldsymbol{x}}=\boldsymbol{F}_{\boldsymbol{a}}{ }^{T} \boldsymbol{J}_{\boldsymbol{a}}{ }^{-T} \boldsymbol{J}_{\boldsymbol{a}}{ }^{-1} \dot{\boldsymbol{X}}_{\boldsymbol{a}}
$$

Because (62) is stable from the assumption, the energy injected to the system should be dissipative.

$$
-\left(C_{f}+1\right) \boldsymbol{F}_{\boldsymbol{a}}{ }^{r e s T} \boldsymbol{J}_{\boldsymbol{a}}{ }^{-T} \boldsymbol{J}_{\boldsymbol{a}}{ }^{-1} \dot{\boldsymbol{X}}_{\boldsymbol{a}}^{\text {res }} \leq 0 .
$$

Similarly, the energy injected to (53) with a force controller (65) is derived in (66).

$$
\begin{aligned}
\boldsymbol{F}_{\boldsymbol{b}}{ }^{\text {input }}= & -C_{f} \boldsymbol{F}_{\boldsymbol{b}}{ }^{\text {res }}+\boldsymbol{M}_{\boldsymbol{b}} \dot{\boldsymbol{J}}_{\boldsymbol{b}} \dot{\boldsymbol{x}}^{\text {res }} \ldots \ldots \ldots \\
& -\left(C_{f}+1\right) \boldsymbol{F}_{\boldsymbol{b}}{ }^{\text {res } T} \boldsymbol{J}_{\boldsymbol{b}}{ }^{-T} \boldsymbol{J}_{\boldsymbol{b}}{ }^{-1} \dot{\boldsymbol{X}}_{\boldsymbol{b}}{ }^{\text {res }}
\end{aligned}
$$

Substituting (50), (51), (54), (55), and (64) for (66), the following inequality is derived.

$$
\begin{aligned}
& -\left(C_{f}+1\right) \boldsymbol{F}_{\boldsymbol{b}}{ }^{r e s T} \boldsymbol{J}_{\boldsymbol{b}}{ }^{-T} \boldsymbol{J}_{\boldsymbol{b}}{ }^{-1} \dot{\boldsymbol{X}}_{\boldsymbol{b}}{ }^{\text {res }}= \\
& -\left(C_{f}+1\right) \boldsymbol{F}_{\boldsymbol{a}}{ }^{r e s T} \boldsymbol{J}_{\boldsymbol{a}}{ }^{-T} \boldsymbol{J}_{\boldsymbol{a}}{ }^{-1} \dot{\boldsymbol{X}}_{\boldsymbol{a}}{ }^{\text {res }} \leq 0
\end{aligned}
$$

Therefore, force control with constraints is also stable.

5.5 Oblique Coordinate Control Because the modal mass matrix of the position constrained bilateral control is a diagonal matrix as shown in (35), the position and force controllers of the position constrained bilateral control are independent. Hence, we can summarize that if the original 4channel bilateral controller is stable, our position constrained bilateral controller, which is a combination of position and force controllers, is also stable.

\section{Experiment}

In this section, the validity of the proposed methods is experimentally verified. The reaction force was estimated by a reaction force observer (RFOB) ${ }^{(31)}$ and disturbances such as friction and modeling errors were eliminated by a DOB. Two kinds of motion: free motion and contact motion to a piece of sponge, were examined for each of the constraints. In the contact motion, a piece of sponge was located in about $0.005[\mathrm{~m}]$ of the slave side. The parameters used in this experiments are shown in Table 1. 
Table 1. Control parameters

\begin{tabular}{|l||l|l|}
\hline$m$ & Actuator Mass & $0.5[\mathrm{~kg}]$ \\
$K_{f}$ & Force Feedback Gain & 3.0 \\
$K_{p}$ & Proportional Feedback Gain & $900\left[1 / \mathrm{s}^{2}\right]$ \\
$K_{v}$ & Derivative Feedback Gain & $60[1 / \mathrm{s}]$ \\
$g$ & Cut-off angular Frequency of DOB & $500[\mathrm{rad} / \mathrm{s}]$ \\
$g_{\text {reac }}$ & Cut-off angular Frequency of RFOB & $500[\mathrm{rad} / \mathrm{s}]$ \\
$S t$ & Sampling Time & $0.0001[\mathrm{~s}]$ \\
$x_{\text {lim }}$ & Position Limitation Value & $0.02[\mathrm{~m}]$ \\
$a$ & Position Threshold Value & $0.01[\mathrm{~m}]$ \\
\hline
\end{tabular}

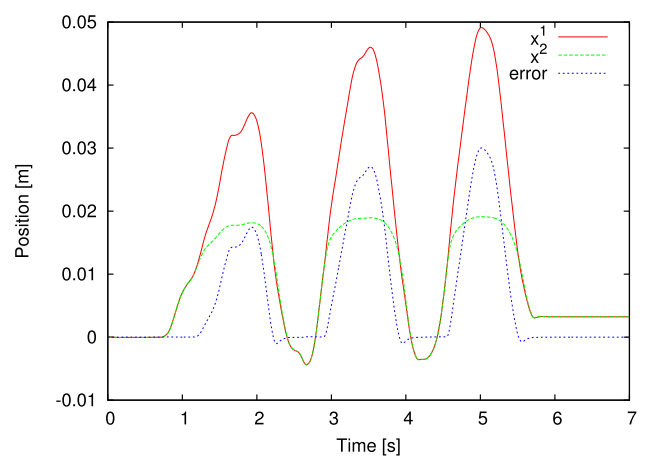

Fig. 5. Position response (Constraint 1, free motion)

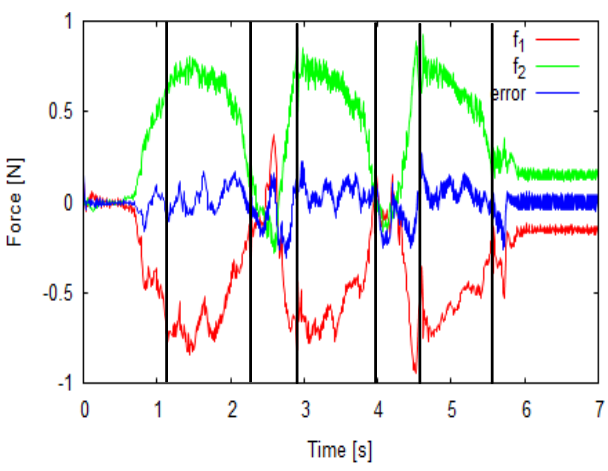

Fig. 6. Force response (Constraint 1, free motion)

Fig. 5 to Fig. 8 are the experimental results of Constraint 1. Fig. 9 to Fig. 12 are those of Constraint 2. Red lines denote the responses of the master, green lines denote the responses of the slave, and the blue lines are the errors, respectively. Vertical lines in the force responses denote the time that the position of the slave passed the threshold $\left(\boldsymbol{x}_{\mathbf{2}}=a\right)$.

From Fig. 5, Fig. 7, Fig. 9, and Fig. 11, the position of the slave did not violate the limitation value $x_{\text {lim }}=0.02$ with all of the cases. These results verified that constrained motion was stably obtained.

From Fig. 5 and Fig. 7, the position responses of the master and the slave were different when the slave went over the threshold $a$. On the other hand, force responses of the robots were almost equal as shown in Fig. 6 and Fig. 8. Therefore, Constraint 1 could give position limitation and force regulation at the same time. In addition, the operator felt as if the environments got softer because the reaction force was not increased even though the operator pushed the master robot.

From Fig. 9 and Fig. 11 position responses of the robots were equal even in the dangerous area $a<x_{2}<x_{\text {lim }}$. On the other hand, force responses of the robots were different in the dangerous area as shown in Fig. 10 and Fig. 12. Position limitation and position tracking were therefore obtained at

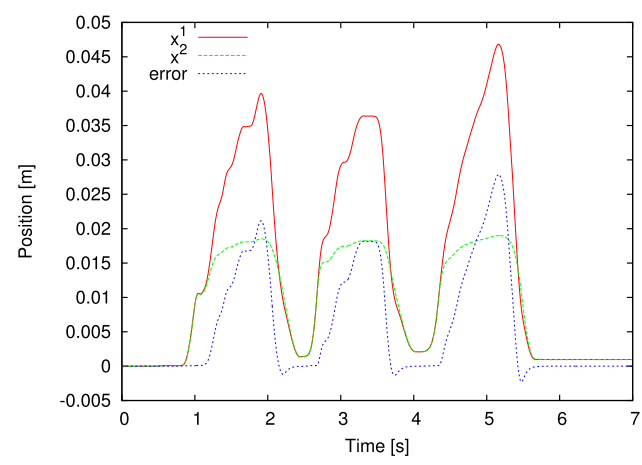

Fig. 7. Position response (Constraint 1, contact motion)

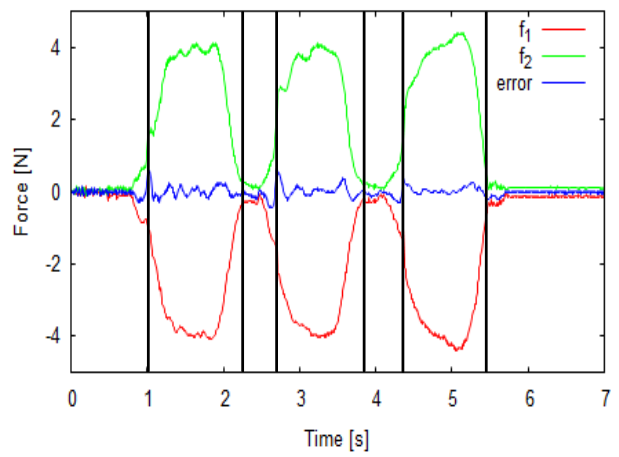

Fig. 8. Force response (Constraint 1, contact motion)

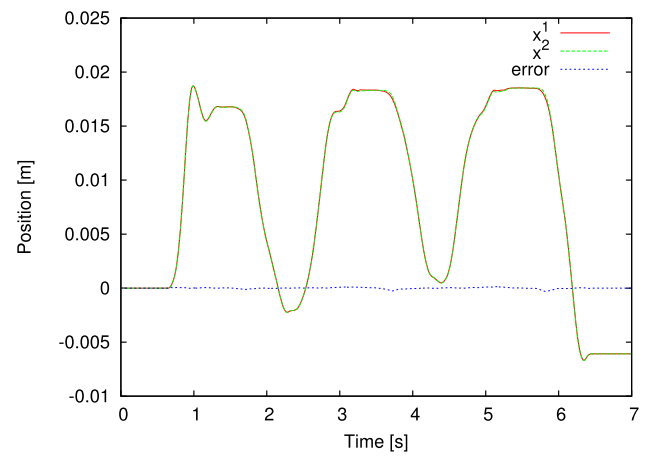

Fig. 9. Position response (Constraint 2, free motion)

the same time by Constraint 2. Even though the operator added large force to the master robot, the position of the master robot was constrained. It means that the operator felt as if there was a wall.

Note that the proposed methods showed small dependency on environments. The reason of this good characteristic is that the DOB powerfully suppressed perturbations of environments and robot models. In addition, we focused on bilateral control between single-DOF robots in this paper. Because the proposed method realizes position constraints by kinetic transform, the constraints are easily implemented to multi-DOF robots by defining a constraint matrix, which is a combination of single-DOF constraint matrices.

\section{Conclusion}

In this paper, two kinds of constrained bilateral controllers were proposed. The first constraint ensures limitation of the slave position and the force regulation. The other ensures limitation of the slave position and the position tracking. Their validity was analytically and experimentally verified, 


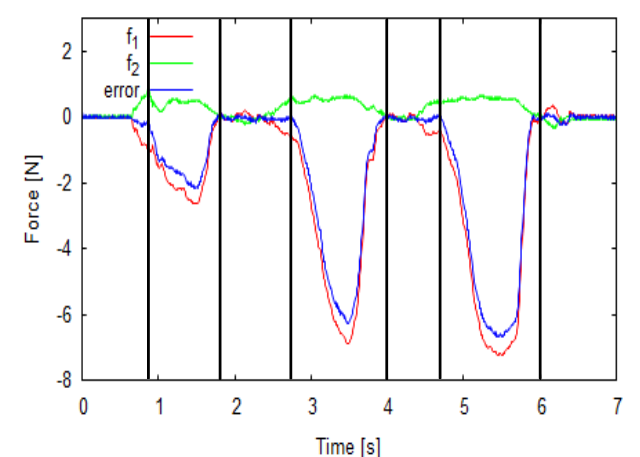

Fig. 10. Force response (Constraint 2, free motion)

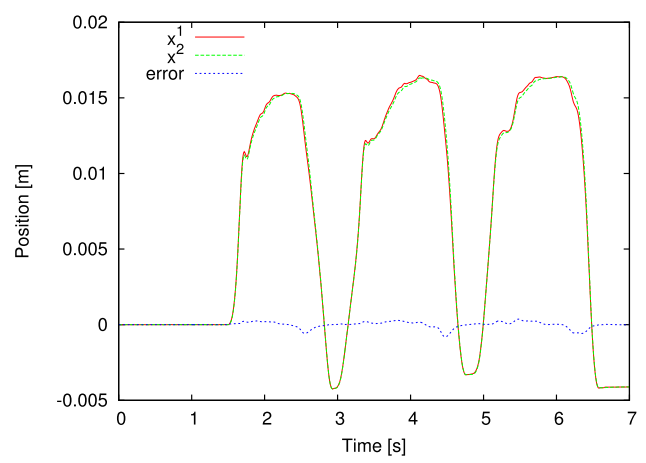

Fig. 11. Position response (Constraint 2, contact motion)

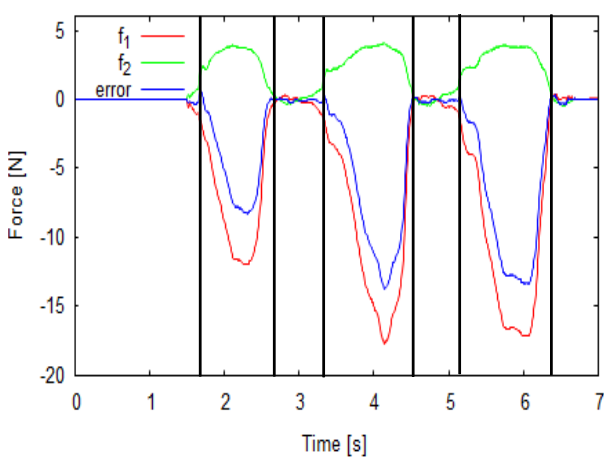

Fig. 12. Force response (Constraint 2, contact motion)

and stable and precise bilateral control was achieved even though the slave position was constrained. These constraints can be adapted to not only bilateral control but also many other robot systems, such as, joint limits of arm robots and obstacle avoidance of mobile robots. However, the proposed method cannot realize actuator saturation and force limits, because the actuator saturation and force limits are functions of force, not position. The proposed method defines constraints by coordinate transform making the constraints functions of position. Therefore, this is still an open problem and we will improve it. As shown in this paper, the proposed method can assure safety without caring the stability, and the designing efforts are drastically reduced. The proposed constraints must be key technologies for human-friendly robot systems.

\section{References}

( 1 ) U. Hagn, T. Ortmaier, R. Konietschke, B. Kubler, U. Seibold, A. Tobergte, M. Nickl, S. Jorg, and G. Hirzinger: "Telemanipulator for remote minimally invasive surgery", IEEE Magazine Robotics and Automation, Vol.15, pp.2838 (2008)

( 2 ) C.-H. King, M.O. Culjat, M.L. Franco, J.W. Bisley, G.P. Carman, E.P. Dutson, and W.S. Grundfest: "A multielement tactile feedback system for robot-assisted minimally invasive surgery", IEEE Trans. Haptics, Vol.2, pp.52-56 (2008)

( 3 ) C.-H. King, M.O. Culjat, M.L. Franco, C.E. Lewis, E.P. Dutson, W.S Grundfest, and J.W. Bisley: "Tactile feedback induces reduced grasping force in robot-assisted surgery", IEEE Trans. Haptics, Vol.2, pp.103-110 (2009)

( 4 ) S.E. Salcudean and J. Yan: "Toward a force-reflecting motion-scaling system for microsurgery", in Proc. of the IEEE International Conference on Robotics and Automation, Vol.3, pp.2296-2301 (1994)

( 5 ) M. Shuzo, H. Arai, R. Kanzaki, and I. Shimoyama: "A nano lead on a force sensing cantilever for bilateral manipulation of a single cell", in Proc. of the IEEE International Conference on Solid-state Sensors, Actuators and Microsystems, Vol.2, pp.1720-1723 (1993)

( 6 ) I. Gaponov, R. Jee-Hwan, C. Seong-Joo, C. Hyun-Chan, and Y. Poduraev: "Telerobotic system for cell manipulation", in Proc. of the IEEE/ASME International Conference on Advanced Intelligent Mechatronics, pp.165-169 (2008)

( 7 ) K. Sung-Gaun and M. Sitti: "Task-based and stable telenanomanipulation in a nanoscale virtual environment", IEEE Trans. Atomation Science and Engineering, Vol.3, pp.240-247 (2006)

( 8 ) K. Natori and K. Ohnishi: "A design method of communication disturbance observer for time-delay compensation, taking the dynamic property of network disturbance into account", IEEE Trans. Industrial Electronics, Vol.55, pp.2152-2168 (2008)

( 9 ) T. Slama, A. Trevisani, D. Aubry, R. Oboe, and F. Kratz: "Experimental analysis of an internet-based bilateral teleoperation system with motion and force scaling using a model predictive controller", IEEE Trans. Industrial Electronics, Vol.55, pp.3290-3299 (2008)

(10) B. Hannaford: "A design framework for teleoperators with kinesthetic feedback", IEEE Trans. Robotics and Automations, Vol.5, No.4, pp.426-434 (1989)

(11) D.A. Lawrence: "Stability and transparency in bilateral teleoperation", IEEE Trans. Robotics and Automation, Vol.9, No.5, pp.624-637 (1993)

(12) R.J. Anderson and M.W. Spong: "Bilateral control of teleoperators with time delay”, IEEE Trans. Automatic Control, Vol.34, No.5, pp.494-501 (1989)

(13) K. Hashtrudi-Zaad and S.E. Salcudean: "Bilateral parallel force/position teleoperation control", Journal of Robotic Systems, Vol.19, No.4, pp.155-167 (2002)

(14) M. Tavakoli, R.V. Patel, and M. Moallem: "A force-reflective master-slave system for minimally invasive surgery", in Proc. of the IEEE/RSJ International Conference on Intelligent Robots and Systems, Vol.4, pp.3077-3082 (2003)

(15) Y. Yokokohji and T. Yoshikawa: "Bilateral control of master-slave manipulators for ideal kinesthetic coupling - formulation and experiment", IEEE Trans. Robotics and Automation, Vol.10, No.5, pp.605-620 (1994)

(16) Y. Matsumoto, S. Katsura, and K. Ohnishi: "An analysis and design of bilateral control based on disturbance observer", in Proc. of the 10th IEEE International Conference on Industrial Technology, pp.802-807 (2003)

(17) K. Ohnishi, M. Shibata, and T. Murakami: "Motion control for advanced mechatronics", IEEE/ASME Trans. Mechatronics, Vol.1, No.1, pp.56-67 (1996)

(18) M.V. Kothare, V. Balakrishnan, and M. Morari: "Robust constrained model predictive control using linear matrix inequalities", Automatica, Vol.32, pp.1361-1379 (1996)

(19) E.I. Verriest and G. Pajunen: "Quadratically saturated regulator for constrained linear systems", IEEE Trans. Automatic Control, Vol.41, pp.992-995 (1996)

(20) N. Kapoor, A.R. Teel, and P. Daoutidis: "An anti-windup design for linear systems with input saturations", Automatica, Vol.34, pp.559-574 (1998)

(21) A. Casavola, E. Mosca, and M. Papini: "Control under constraints: An application of the command governor approach to an inverted pendulum", IEEE Trans. Control Systems Technology, Vol.12, pp.193-204 (2004)

(22) T. Keiichi, S. Hyodo, and K. Ohnishi: "A design method of autonomous hazard avoidance controller with selected ratio in bilateral teleoperation", in Proc. of 10th IEEE International Workshop on Advanced Motion Control, pp.604-612 (2008)

(23) A. Casavola and M. Sorbara: "Towards constrained teleoperation for safe long-distance robotics surgical operations", in Proc. of the 2005 IEEE Inter- 
national Conference on Robotics and Automation, pp.685-690 (2005)

(24) S. Sakaino, T. Sato, and K. Ohnishi: "Multi-dof micro macro bilateral controller using oblique coordinate control", IEEE Trans. Industrial Informatics, Vol.7, pp.446-454 (2011)

(25) S. Sakaino, T. Sato, and K. Ohnishi: "Task hierarchy for position limitation and bilateral control by oblique coordinate control", in Proc. of the 35th Annual Conference of the IEEE Industrial Electronics, pp.1808-1813 (2009)

(26) K. Natori, S. Katsura, T. Tsuji, and K. Ohnishi: "Analysis of bilateral systems with time delay", in Proc. of International Symposium on Industrial Electronics, ISIE 2005, pp.1511-1516 (2005)

(27) K. Natori, R. Oboe, and K. Ohnishi: "Stability analysis and practical design procedure of time delayed control systems with communication disturbance observer", IEEE Trans. Industrial Informatics, Vol.4, pp.185-197 (2008)

(28) U. Tumerdem and K. Ohnishi: " $\mathrm{L}_{2}$ stability analysis of four channel teleoperation and experiments under varying time delay", in Proc. of the 11th IEEE International Workshop on Advanced Motion Control, pp.643-648 (2010)

(29) S. Sakaino, T. Sato, and K. Ohnishi: "Precise position/force hybrid control with modal mass decoupling and bilateral communication between different structures", IEEE Trans. Industrial Informatics, Vol.7, No.2, pp.266-276 (2011)

(30) R. Kubo, T. Shimono, and K. Ohnishi: "A bilateral controller design method for master-slave robots with different dof", in Proc. of IEEE International Conference on Industrial Technology 2006, pp.328-333 (2006)

(31) T. Murakami, F. Yu, and K. Ohnishi: "Torque sensorless control in multidegree-of-freedom manipulator", IEEE Trans. Industrial Electronics, Vol.40, No.2, pp.259-265 (1993)

Sho Sakaino (Member) received the B.E. degree in system design

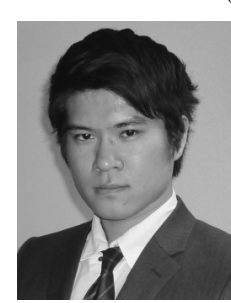
engineering and the M.E. and Ph.D. degrees in integrated design engineering from Keio University, Yokohama, Japan, in 2006, 2008, and 2011, respectively. He is currently an assistant professor with the Department of Electrical and Electronic Systems, Saitama University, Saitama, Japan. His research interests include mechatronics, motion control, robotics, and haptics. He received IEEJ Industry Application Society Distinguished Transaction Paper
Tomoya Sato (Non-member) received the B.E. degree in system de-

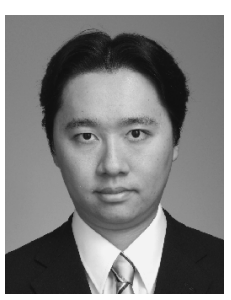
sign engineering and the M.E. and Ph.D. degrees in integrated design engineering from Keio University, Yokohama, Japan, in 2007, 2009, and 2010, respectively. He is currently working for Sony Computer Entertainment Inc. His research interests include biped robots and haptics.

Kouhei Ohnishi

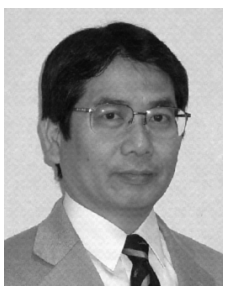

(Fellow) received the B.E., M.E., and Ph.D. degrees in electrical engineering from the University of Tokyo, Tokyo, Japan, in 1975, 1977 and 1980, respectively. Since 1980, he has been with Keio University, Yokohama, Japan. His research interests include mechatronics, motion control, robotics, and haptics.

Award in 2011 